\title{
Plasmonic nanoparticle enhanced light absorption in GaAs solar cells
}

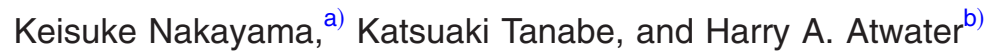 \\ Thomas J. Watson Laboratory of Applied Physics, California Institute of Technology, Pasadena, California \\ 91125, USA
}

(Received 24 July 2008; accepted 31 August 2008; published online 22 September 2008)

\begin{abstract}
We demonstrate an improvement in efficiency of optically thin GaAs solar cells decorated with size-controlled Ag nanoparticles fabricated by masked deposition through anodic aluminum oxide templates. The strong scattering by the interacting surface plasmons in densely formed high aspect-ratio nanoparticles effectively increases the optical path of the incident light in the absorber layers resulting in an $8 \%$ increase in the short circuit current density of the cell. The nanoparticle array sheet conductivity also reduces the cell surface sheet resistance evidenced by an improved fill factor. This dual function of plasmonic nanoparticles has potential to enable thinner photovoltaic layers in solar cells. (C) 2008 American Institute of Physics. [DOI: 10.1063/1.2988288]
\end{abstract}

High efficiency solar cell design requires optically thick photovoltaic layers to enable nearly complete light absorption, as well as minority carrier diffusion lengths several times the material thickness to allow efficient carrier collection. However, increased cell efficiency and reduced material utilization both motivate a reduction in cell active region thickness provided the optical thickness can be maintained. An attractive approach involves plasmonic nanostructures. In recent years, plasmonic nanoparticles closely coupled to absorbing semiconductors have been utilized to enhance absorption in ultrathin film solar cells. ${ }^{1-5}$ The antennalike nanoparticle response caused by dipole oscillation of localized surface plasmons serves to increase the material extinction for incident light owing to an enhanced local electromagnetic field near the nanoparticles at the surface plasmon resonance as well as an enhanced scattering cross section for offresonant light. From the perspective of photovoltaic conversion, plasmon resonant absorption represents an unwanted loss process, but off-resonant enhanced scattering can yield increases in absorption and photocurrent collection. Since the optical property of the plasmonic nanoparticles sensitively depends on nanoparticle size and shape, ${ }^{6}$ proper morphological design of the nanoparticles is a requirement for achieving high efficiency plasmonic solar cells. Anodic aluminum oxide (AAO) can serve as an evaporation mask for metal deposition to form size-controlled nanoparticle array on a substrate. ${ }^{7}$ We have reported great flexibility in nanoparticle array synthesis on optically thin GaAs solar cells by masked deposition through AAO templates and examined the size effects of hemispherical metal nanoparticle arrays ${ }^{8,9} \mathrm{In}$ this letter we describe the photocurrent enhancements of the GaAs solar cells by $110 \mathrm{~nm}$ diameter $\mathrm{Ag}$ nanoparticles formed on. The shape and density of the nanoparticles are uniformly and systematically controlled using AAO templates. The optical property of the nanoparticle arrays as well as the photovoltaic property of the cells decorated by the nanoparticle arrays is discussed.

AAO templates were fabricated through a two-step anodization of $4 \mathrm{~N}$-grade aluminum sheets. Anodization was carried out in two conditions to obtain the templates with

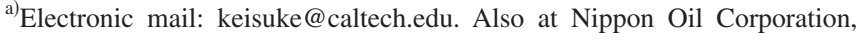
Yokohama 231-0815, Japan.

${ }^{b)}$ Electronic mail: haa@caltech.edu.
}

different nanopore densities: at $80 \mathrm{~V}$ in a mixture of $0.3 \mathrm{~mol} \mathrm{l}^{-1}$ oxalic acid and $0.3 \mathrm{~mol}^{-1}$ malonic acid solutions, and at $120 \mathrm{~V}$ in a $0.3 \mathrm{~mol} \mathrm{l}^{-1}$ malonic acid solution. ${ }^{10}$ Afterwards, the aluminum sheet was selectively removed in saturated iodine solution in methanol, and the bottom layer of the oxide was chemically etched in 5\% dilute solution of phosphoric acid (85\%) to form a through-hole structure. The nanopore diameter in the templates could be increased with the chemical etching time and decreased by evaporating $\mathrm{Ag}$ onto the templates prior to the use as the evaporation mask. The insets in Figs. 1(a) and 1(d) show the scanning electron microscope (SEM) images of two AAO templates in which the nanopores have the average diameter of $110 \mathrm{~nm}$ with the densities of $3.3 \times 10^{9}$ and $1.8 \times 10^{9} \mathrm{~cm}^{-2}$, respectively. The through-hole AAO template was then placed directly onto the window layer of the GaAs solar cell. Thermal evaporation of $\mathrm{Ag}$ through the AAO templates resulted in formation of dense and sparse arrays of $\mathrm{Ag}$ nanoparticles on GaAs solar cells which possess the same diameters and interparticle spacing as the nanopores in the AAO templates [Figs. 1(a) and 1(d)]. The height of the nanoparticles can be controlled simply with the deposition thickness [Figs. 1(b), 1(c), 1(e), and 1(f)]. The nanoparticles change their shape from hemispheres to cones as the heights are increased, because the nanopore diameter in the AAO templates is gradually de-

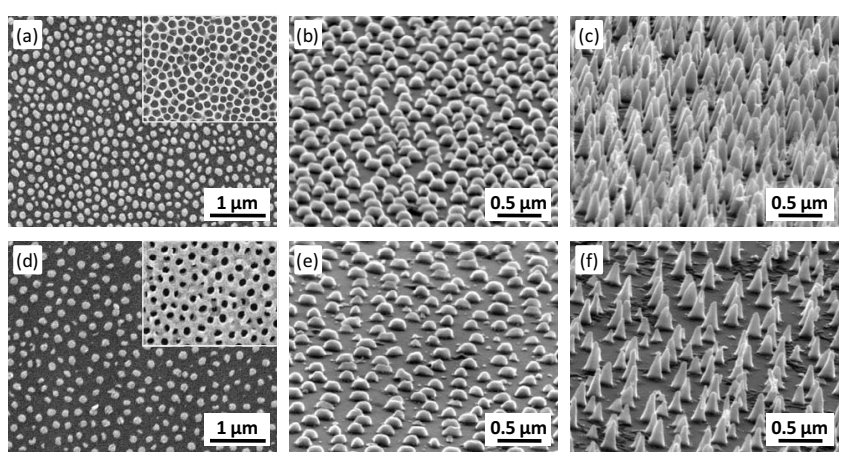

FIG. 1. SEM images of [(a)-(c)] dense and [(d)-(f)] sparse arrays of asdeposited $110 \mathrm{~nm}$ diameter $\mathrm{Ag}$ nanoparticles. The insets of (a) and (d) are corresponding AAO templates fabricated at 80 and $120 \mathrm{~V}$. Array density and nanoparticle average height are (b) $3.3 \times 10^{9} \mathrm{~cm}^{-2}$ density $/ 55 \mathrm{~nm}$ height (DL), (c) $3.3 \times 10^{9} \mathrm{~cm}^{-2}$ density/220 nm height (DH), (e) $1.8 \times 10^{9} \mathrm{~cm}^{-2}$ density $/ 55 \mathrm{~nm}$ height (SL), and (f) $1.8 \times 10^{9} \mathrm{~cm}^{-2}$ density $/ 220 \mathrm{~nm}$ height $(\mathrm{SH})$; the images of which were taken from the angle of $75^{\circ}$. 

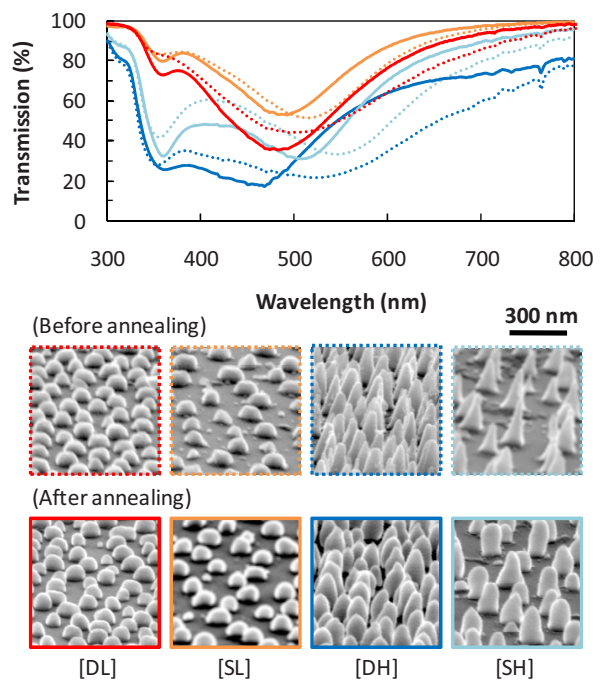

FIG. 2. (Color online) Extinction spectra for $\mathrm{Ag}$ nanoparticles on glass substrates before (dot lines) and after annealing at $200{ }^{\circ} \mathrm{C}$ (solid lines) corresponding the SEM images below.

creased due to the Ag deposition onto the masks.

The Ag nanoparticle shape can also be tuned by a thermal annealing at temperatures well below the melting point of Ag. ${ }^{11}$ Annealing the $\mathrm{Ag}$ nanoparticles at $200{ }^{\circ} \mathrm{C}$ for $30 \mathrm{~min}$ in $5 \% \mathrm{H}_{2}$ diluted by $\mathrm{N}_{2}$ induced surface melting migration resulting in the restructured $\mathrm{Ag}$ nanoparticles which have more hemispherical shapes relative to those immediately after deposition (Fig. 2). These morphological changes are seen in the extinction spectra for the nanoparticles formed on glass substrates. The extinction peaks in Fig. 2 in the visible region correspond to the surface plasmon resonance in $\mathrm{Ag}$ nanoparticles. The relatively sharp absorption at
$360 \mathrm{~nm}$ is related to electronic interband transitions in Ag. ${ }^{12}$ Annealing the nanoparticles yielded blueshifted surface plasmon resonances, which are attributed to the improvements in the symmetry of the nanoparticle shapes. ${ }^{6,13}$ After annealing, both the dense and sparse arrays of lower aspect-ratio nanoparticles have the peaks at similar wavelength, while the dense array of higher aspect-ratio nanoparticles has blueshifted peak relative to the sparse array, implying the existence of strong electromagnetic interactions in the higher aspect-ratio nanoparticles formed in dense. ${ }^{14}$

Here we investigated the effects of these four arrays, densely formed low (DL) nanoparticles, sparsely formed low (SL) nanoparticles, densely formed high (DH) nanoparticles, and sparsely formed high (SH) nanoaprticles, on the photocurrent response of the optically thin GaAs solar cell, as shown in Fig. 3(a). Our cell design employed in these experiments is a prototype designed to clearly investigate the effects of plasmonic scattering. Thus a GaAs solar cell, in which the photovoltaic layers were intentionally designed to be much thinner than the absorption length, was grown on (100) $n$-GaAs substrate by metal organic chemical vapor deposition with $\mathrm{Zn}$ as the $p$-type dopant and $\mathrm{Si}$ as the $n$-type dopant. The thickness of $p-n$ GaAs photovoltaic layers is $200 \mathrm{~nm}$, which is less than $10 \%$ of the photovoltaic layer thickness in conventional high performance GaAs cells. ${ }^{15}$ In addition, a high band gap back surface field (BSF) layer of $n-\mathrm{Al}_{0.8} \mathrm{Ga}_{0.2} \mathrm{As}$ is formed to prevent carrier generation in this layer. ${ }^{16}$ Half of the light above the band gap of GaAs is absorbed by the $p-\mathrm{Al}_{0.8} \mathrm{Ga}_{0.2} \mathrm{As}$ window and $p$ - $n$ GaAs photovoltaic layers and the remaining light transmitted through the layers is absorbed by the underlying GaAs without carrier collection. After both front and bottom Au contacts were formed, the GaAs cap layer was selectively etched in 4: 1

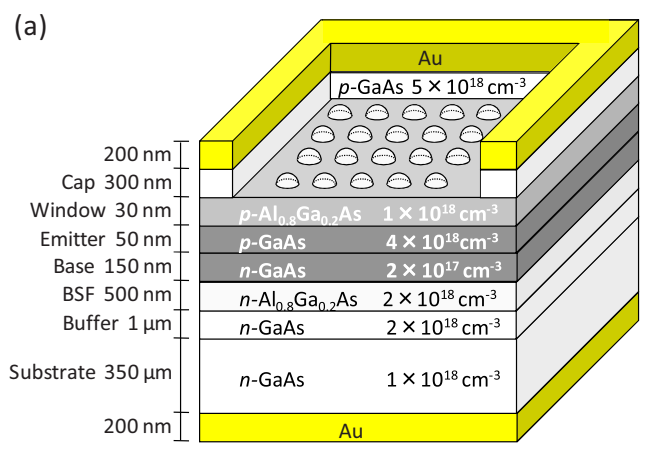

(b)

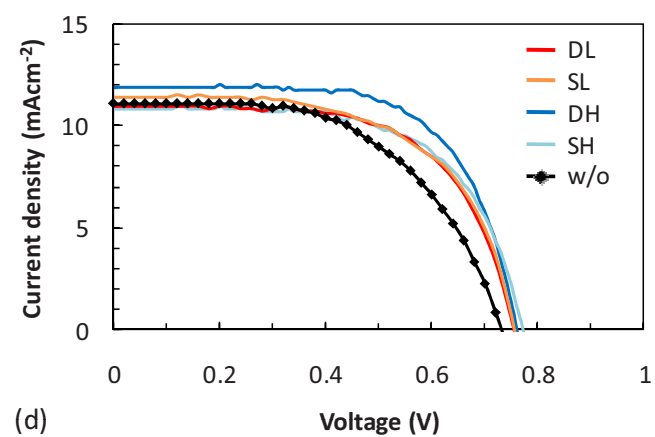

(c)
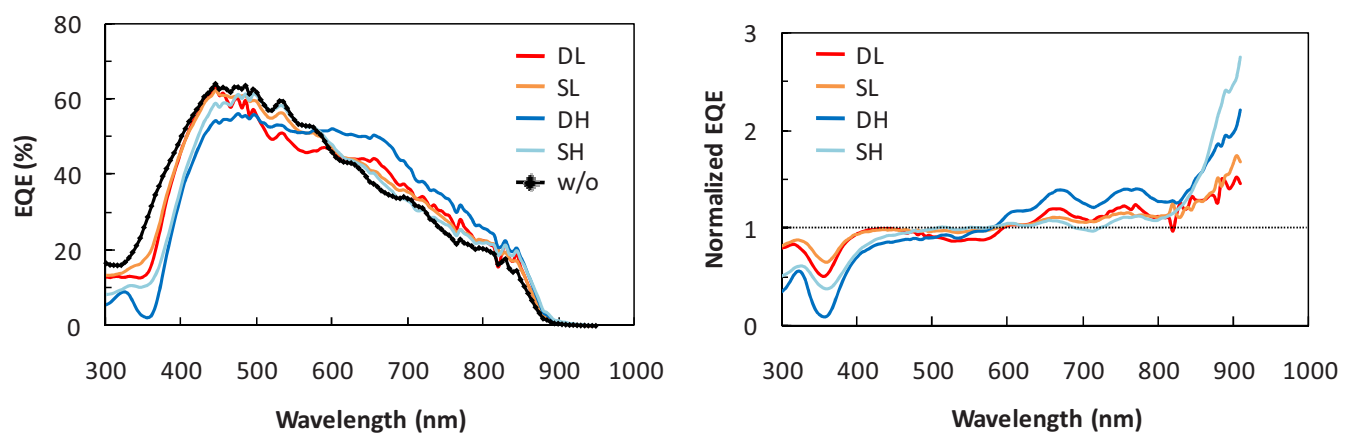

FIG. 3. (Color online) (a) A layer structure of the GaAs solar cell with the area of $6 \times 7 \mathrm{~mm}^{2}$ and the top contact linewidth of $1 \mathrm{~mm}$. Photocurrent generates in the top three layers. The through-hole AAO template about $4 \times 4 \mathrm{~mm}^{2}$ in size was placed onto the cell to form the nanoparticle array. In the photovoltaic measurements, the exposed area of the cell was limited to $3 \times 3 \mathrm{~mm}^{2}$ using a metal mask. (b) Photovoltaic $I$ - $V$ curves for the GaAs solar cells under one-sun illumination (AM1.5, $100 \mathrm{~mW} \mathrm{~cm}{ }^{-2}$ ) using a solar simulator. (c) Photovoltaic spectral responses for the GaAs solar cells measured by shining monochromatic light from a $300 \mathrm{~W}$ xenon lamp. (d) Normalized spectral responses for the nanoparticle-decorated GaAs solar cells by the spectrum for the reference cell. 
TABLE I. $I-V$ characteristics of the GaAs solar cells.

\begin{tabular}{|c|c|c|c|c|c|c|c|}
\hline \multirow[b]{2}{*}{$\mathrm{Ag}$ array } & \multicolumn{3}{|c|}{ Designed values } & \multirow[b]{2}{*}{$J_{\mathrm{sc}}\left(\mathrm{mA} \mathrm{cm}^{-2}\right)$} & \multirow[b]{2}{*}{$V_{\mathrm{oc}}(\mathrm{V})$} & \multirow[b]{2}{*}{ FF } & \multirow[b]{2}{*}{$\eta(\%)$} \\
\hline & Diameter (nm) & Height (nm) & Density $\left(\mathrm{cm}^{-2}\right)$ & & & & \\
\hline DL & 110 & 55 & $3.3 \times 10^{9}$ & 10.9 & 0.76 & 0.62 & 5.1 \\
\hline SL & & & $1.8 \times 10^{9}$ & 11.4 & 0.75 & 0.60 & 5.1 \\
\hline DH & & 220 & $3.3 \times 10^{9}$ & 11.9 & 0.76 & 0.65 & 5.9 \\
\hline SH & & & $1.8 \times 10^{9}$ & 10.8 & 0.77 & 0.64 & 5.3 \\
\hline $\mathrm{W} / \mathrm{o}$ & N/A (reference) & & & 11.0 & 0.73 & 0.55 & 4.7 \\
\hline
\end{tabular}

citric acid (50\%): $\mathrm{H}_{2} \mathrm{O}_{2}(30 \%)$ solution, ${ }^{17}$ followed by $\mathrm{Ag}$ deposition through AAO template and annealing at $200{ }^{\circ} \mathrm{C}$ for $30 \mathrm{~min}$ in $5 \% \mathrm{H}_{2}$ diluted by $\mathrm{N}_{2}$.

The photovoltaic properties of the GaAs solar cells are given in Table I and Figs. 3(b)-3(d). The arrays had great influence on the photocurrent of the cells, in which the best photocurrent enhancement was induced by the DH nanoparticles with an $8 \%$ increase in the short circuit current density $\left(J_{\mathrm{sc}}\right)$. To understand the observed photocurrent, the external quantum efficiencies (EQEs) of the nanoparticle-decorated cells in Fig. 3(c) are normalized by that of the reference cell [Fig. 3(d)]. All the nanoparticle-decorated cells have the decreased photocurrent responses shorter than around $600 \mathrm{~nm}$ where the surface plasmon resonance effects show up more strikingly, while the cells have the enhanced photocurrent responses at longer wavelengths. The photocurrent loss/gain relative to the reference cell is $-9 \% / 12 \%$ for the DL cell, $-4 \% / 10 \%$ for the SL cell, $-14 \% / 26 \%$ for the DH cell, and $-7 \% / 7 \%$ for the SH cell, respectively, calculated from the EQEs under the AM $1.5 \mathrm{G}$ illumination. The sparse arrays enable the reduction in the loss of incident light resulting from either absorption or backscattering by the nanoparticles near the surface plasmon resonances, while the plasmonic scattering can be enhanced in the dense array cases at wavelengths below the surface plasmon resonances. Especially the DH nanoparticles significantly enhances the photocurrent, which can be attributed to the strong scattering by the interacting surface plasmons.In previous reports, plasmonic nanoparticle arrays were formed by either annealing thin metal film or dispersing nanoparticle-containing solution and thus the nanoparticle size, shape, and density could not be uniformly and systematically controlled, which means that the plasmonic scattering could be induced by both the individual nanoparticle and interacting nanoparticles. Our results demonstrate that the arrangement of the nanoparticles with the near-field coupling between the nanoparticles, which causes the increase in the nanoparticle coverage on the solar cell surface, enhances the plasmonic scattering and thus in turn increases the absorption of the incident light.

Because of the absorbing GaAs substrate, however, the cell design is not optimum for overall photocurrent enhancement; the present cell structure potentially gives rise to only a slight gain in overall $J_{\text {sc }}$ due to the single optical path in the photovoltaic layers. Further photocurrent enhancement can be obtained by combining plasmonic nanoparticles with a thin GaAs cell that has greater refractive index contrast relative to a low index substrate layer, to provide for total internal reflection at the cell back interface and guided wave propagation in the cell photovoltaic layers.

Another advantage of nanoparticle arrays is in apparent in the improvements in the fill factor (FF) for the cells, indi- cating the reduction in the surface sheet resistance. This effect can be expected in all solar cells that form Ohmic contact between the nanoparticle array and the cell window layer. This suggests, for example, that in even optically thick cells, plasmonic nanoparticles could be employed to serve as transparent conductors to increase the FF-and in turn cell efficiency - for thin lightly doped top window layers, though they do not increase the photocurrent.

In conclusion, we investigated the effects of plasmonic scattering on absorption and photocurrent collection in the prototype GaAs solar cells decorated with size-controlled Ag nanoparticles. At wavelengths below surface plasmon resonance, scattered incident light by the nanoparticles yields enhanced absorption in the photovoltaic layers of the cell The interacting surface plasmons between the nanoparticles can enhance the plasmonic scattering effect. Such cells serve as a model system for investigating plasmon-enhanced absorption in solar cells and enable us to develop guiding principles for solar cell design.

This work was supported by the Department of Energy, Basic Energy Sciences under Grant No. DE-FG0207ER46405, and Nippon Oil Corporation. We also acknowledge support from the Center for Science and Engineering of Materials (CSEM), a NSF Material Research Science and Engineering Center at Caltech, for facilities use.

${ }^{1}$ H. R. Stuart and D. G. Hall, Appl. Phys. Lett. 69, 2327 (1996)

${ }^{2}$ H. R. Stuart and D. G. Hall, Appl. Phys. Lett. 73, 3815 (1998).

${ }^{3}$ D. M. Schaadt, B. Feng, and E. T. Yu, Appl. Phys. Lett. 86, 063106 (2005).

${ }^{4}$ S. Pillai, K. R. Catchpole, T. Trupke, and M. A. Green, J. Appl. Phys. 101, 093105 (2007).

${ }^{5}$ B. P. Rand, P. Peumans, and S. R. Forrest, J. Appl. Phys. 96, 7519 (2004).

${ }^{6}$ C. F. Bohren and D. R. Huffman, Absorption and Scattering of Light by Small Particles (Wiley, New York, 1983).

${ }^{7}$ H. Masuda and M. Satoh, Jpn. J. Appl. Phys., Part 2 35, L126 (1996).

${ }^{8}$ K. Tanabe, K. Nakayama, and H. A. Atwater (unpublished).

${ }^{9}$ K. Nakayama, K. Tanabe, and H. A. Atwater, Proc. SPIE 7047, 704708 (2008)

${ }^{10}$ S. Ono, M. Saito, and H. Asoh, Electrochim. Acta 51, 827 (2005).

${ }^{11}$ P. Royer, J. P. Goudonnet, R. J. Warmack, and T. L. Ferrell, Phys. Rev. B 35, 3753 (1987).

${ }^{12}$ G. Fuster, J. M. Tyler, N. E. Brener, J. Callaway, and D. Bagayoko, Phys. Rev. B 42, 7322 (1990).

${ }^{13}$ J. S. Biteen, N. S. Lewis, and H. A. Atwater, Appl. Phys. Lett. 88, 131109 (2006).

${ }^{14}$ S. A. Maier and H. A. Atwater, J. Appl. Phys. 98, 011101 (2005).

${ }^{15}$ S. P. Tobin, S. M. Vernon, C. Bajgar, L. M. Geoffroy, C. J. Keavney, M. M. Sanfacon, and V. E. Haven, Sol. Cells 24, 103 (1988).

${ }^{16}$ D. E. Aspnes, S. M. Keiso, R. A. Logan, and R. Bhat, J. Appl. Phys. 60, 754 (1986).

${ }^{17}$ J.-H. Kim, D. H. Lim, and G. M. Yang, J. Vac. Sci. Technol. B 16, 558 (1998). 